\title{
Al-doped ZnO films deposited by magnetron sputtering: effect of sputtering parameters on the electrical and optical properties
}

\author{
QINGTAO PAN ${ }^{1,2, *}$, XIN SONG ${ }^{2}$ \\ ${ }^{1}$ Shahe Institute for Glass Technology Research, Xingtai, 054100, China \\ ${ }^{2}$ Baoding Tianwei Solar films Co., Ltd., Baoding 071051, China
}

\begin{abstract}
Aluminum-doped zinc oxide (AZO) thin films were prepared by magnetron sputtering method. The influences of deposition pressure, substrate temperature, Ar flow rate and film thickness on optical and electrical properties were investigated using ultraviolet-visible (UV-Vis) spectrometer and Hall measurements. The experimental results revealed that a low resistivity, smaller than $4 \times 10^{-4} \Omega \cdot \mathrm{cm}$, was obtained when the deposition pressure was smaller than $0.67 \mathrm{~Pa}$ and substrate temperature about $200^{\circ} \mathrm{C}$. Ar flow rate had a small influence on the resistivity but a big influence on the transparency at near infrared range (NIR). We obtained optimized AZO thin films with high conductivity and transparency at low deposition pressure, small Ar flow and appropriate temperature (around $200{ }^{\circ} \mathrm{C}$ ). The etching behavior of the AZO thin films deposited at the different Ar flow rates was also studied in this paper. The results show that Ar flow rate is a very important factor affecting the etching behavior.
\end{abstract}

Keywords: AZO thin films; optical and electrical properties; morphology

\section{Introduction}

Transparent conductive oxides (TCOs) are widely used in a variety of optoelectronic devices, such as transparent thin film transistors and thin film solar cells [1]. Now, indium tin oxide (ITO) film is a very popular choice for TCO materials. However, indium is very rare in the world, so it is very urgent to find its replacement.

Zinc oxide $(\mathrm{ZnO})$ doped with $\mathrm{Al}$ is the most promising alternative material for TCO because of its low resistivity, high transparency, [2-4] and even the low cost. Until now, many techniques have been used for preparing the AZO films, such as magnetron sputtering [5], sol-gel [6, 7], evaporation [8], pulsed laser deposition (PLD) [9], etc. Among these techniques, magnetron sputtering is the most commonly used due to the possibility of fabricating uniform films on different large-area substrates at moderate deposition parameters. Furthermore, sputtering is compatible with the existing

*E-mail: qtpan00@hotmail.com microelectronic/photovoltaic industries and it can be easily transferred to the industrial applications. Low electrical resistivity and high optical transmission are the primary requirements for the TCO films, which depend strongly on the sputtering conditions, such as RF power, sputtering pressure, Ar flow rate, etc. In addition, a textured TCO surface is required to provide an effective light trapping for the application in the thin film solar cells.

In this paper, we investigated the effect of the deposition parameters on the electrical and optical properties of AZO films prepared by RF magnetron sputtering method. Furthermore, we also investigated the etching behavior of the AZO films deposited at different Ar flow rates. An AZO (2 wt.\% $\left.\mathrm{Al}_{2} \mathrm{O}_{3}\right)$ target was used and all the films were deposited on $3.3 \mathrm{~mm}$ thick low iron float glass substrates.

\section{Experimental}

Fig. 1 illustrates a schematic drawing of the magnetron sputtering system that we used in our 
lab. All films were deposited in the high vacuum (base pressure of $\sim 10^{-5} \mathrm{~Pa}$ ) RF sputtering system with $5 \times 28 \mathrm{~cm}^{2}$ planar AZO (2 wt. $\left.\% \mathrm{Al}_{2} \mathrm{O}_{3}\right)$ target. The distance between substrate and cathode was approximately $5 \mathrm{~cm}$. The substrate with the size of $15.6 \mathrm{~cm} \times 15.6 \mathrm{~cm}$ oscillated over the target on the oscillation track. Unless otherwise specified, the applied discharge power on the cathodes was $250 \mathrm{~W}$. The substrate temperature was in the range of $25^{\circ} \mathrm{C}$ to $250{ }^{\circ} \mathrm{C}$, deposition pressure was in the range of $0.4 \mathrm{~Pa}$ to $4 \mathrm{~Pa}$, and the Ar flow was in the range of 2 SCCM to 20 SCCM (SCCM standard cubic centimeters per minute). Argon gas with the purity of $5 \mathrm{~N}$ was used in all experiments.

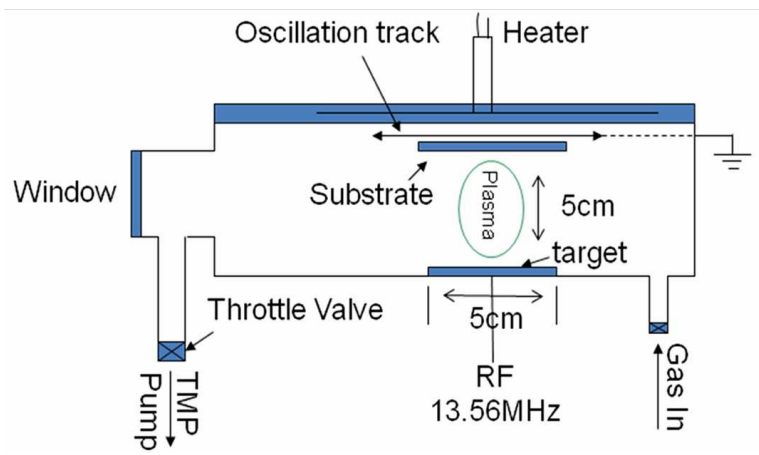

Fig. 1. Schematic representation of the sputtering system.

The electrical properties of the films were investigated by four point probe and Hall-effect measurement using van der Pauw geometry, at room temperature. The film thicknesses were measured with Filmetrics F20 Thin-film Analyzer. The optical properties of the films, such as total transmission (TT), diffuse transmission (DT), haze (defined by the ratio of DT to TT) and reflection as well as absorption were investigated with an UV-Vis-NIR spectrometer (PerkinElmer Lambda 950). The film surface analysis was done using an atomic force microscopy (AFM) (SPI3800N). The film structure was studied by X-ray diffraction measurement (Rigaku RINT2400 with $\mathrm{CuK} \alpha$ radiation). The morphologies were investigated using a field emission scanning electron microscope (FE-SEM, BACPCS4800).

\section{Results and discussion}

\subsection{Influence of deposition pressure on the characteristics of AZO films}

Fig. 2 shows the dependence of the deposition rates and electrical properties on deposition pressure. The deposition rates are almost constant (ca $14 \mathrm{~nm} / \mathrm{min}$ ), when the working pressure is smaller than $0.4 \mathrm{~Pa}$ and then decrease with increasing deposition pressure. When the pressure is of $4.0 \mathrm{~Pa}$, the deposition rate is $7.5 \mathrm{~nm} / \mathrm{min}$, which is only a half of the deposition rate at low pressure. Such behavior is mainly due to the discharge voltage decreasing with the increasing pressure. In accordance with the decreased discharge voltage, the deposition rate also decreases. At high pressures, the low deposition rates are caused by shielding effects of gas particles between the target and substrate.

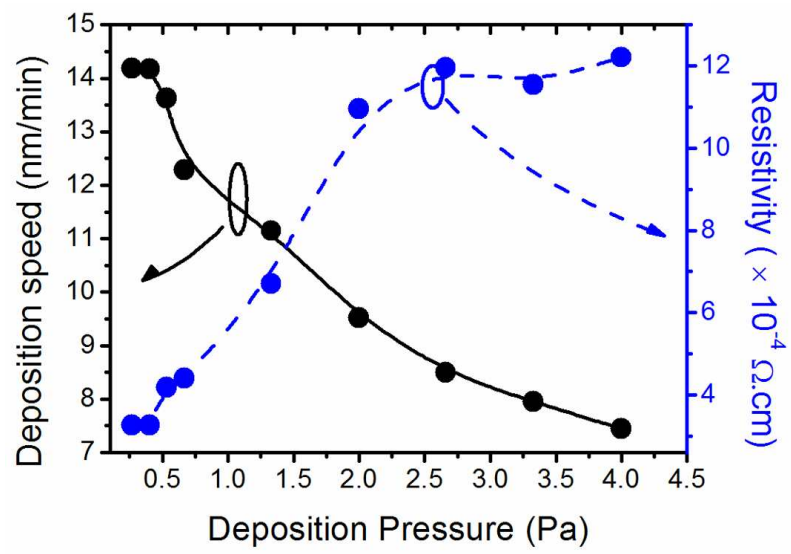

Fig. 2. Deposition rates and resistivity as a function of deposition pressure.

Low resistivity $\left(<5 \times 10^{-4} \Omega \cdot \mathrm{cm}\right)$ could be achieved in the low pressure regime $(\leqslant 0.67 \mathrm{~Pa})$, but it increases with increasing pressure, what is very clearly observed for the RF sputtered films [10]. When the pressure is higher than $2.0 \mathrm{~Pa}$, the resistivity is prone to saturate at the level of $11.5 \times 10^{-4} \Omega \cdot \mathrm{cm}$. Kluth et al. [10] suggested that the increase in resistivity results from a strong decrease in carrier mobility if a certain deposition pressure is exceeded because of the loose structure at high pressure. 


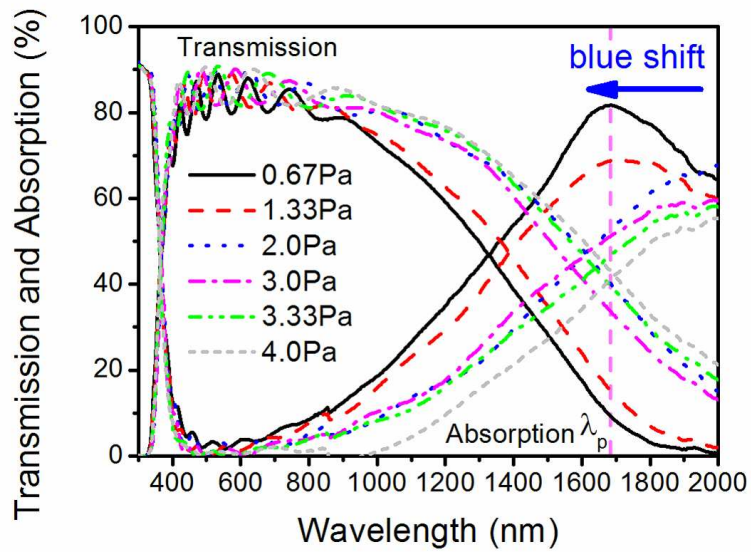

Fig. 3. Transmittance and absorption of the films deposited at different working pressures (film thickness $d=600 \mathrm{~nm}$ to $900 \mathrm{~nm}, \lambda_{\mathrm{p}}$ plasma resonance wavelength).

The transmission and absorption properties of the films are shown in Fig. 3. All the films are highly transparent. The absorption at the near infrared range (NIR) is primarily due to free carriers, and the absorption peak in Fig. 3 is due to the plasma resonance. The plasma resonance wavelength $\left(\lambda_{\mathrm{p}}\right)$ can be given by [1]:

$$
\lambda_{p}=2 \pi c\left(\frac{\varepsilon_{\infty} \varepsilon_{0} m^{*}}{N e^{2}}\right)^{1 / 2}
$$

where $\epsilon_{\infty}$ is the high frequency dielectric constant, $\epsilon_{0}$ is vacuum dielectric constant, $\mathrm{m}^{*}$ is the electron effective mass, e is the electronic charge, $\mathrm{c}$ is the light velocity and $\mathrm{N}$ is free carrier density. In addition, we can find the free carrier density decreases with the working pressure increase. At high working pressure $(\geqslant 2.66 \mathrm{~Pa})$, the plasma resonance peaks are almost at the same wavelength, what means that the carrier densities are almost the same. In the $\mathrm{Al}$ doped $\mathrm{ZnO}$, the carrier density is mainly attributed to (a) deviation from stoichiometry and (b) the doping effect. For the same doping concentration of 2 wt. $\% \mathrm{Al}_{2} \mathrm{O}_{3}$, substrate temperature and deposition power, we can deduce that the carrier density from the $\mathrm{Al}$ impurity is almost the same. For the same Ar flow rate, at high deposition pressure, which means lower pump rate, the $\mathrm{O}$ atom lifetime becomes a little longer, so the $\mathrm{O}: \mathrm{Zn}$ ratio probably increases at high working pressure.
The increase in O:Zn ratio at high working pressure can lead to a change in $\mathrm{O}$ incorporation into the film through the grain boundaries which consequently causes a decrease in carrier concentration [2]. The carrier density decrease with the working pressure increase is the main reason for the increase in resistivity.

\subsection{Influence of thin film thickness on the characteristics of AZO films}

Fig. 4a shows the electrical properties of the films with different thicknesses. The resistivities are all smaller than $4 \times 10^{-4} \Omega \cdot \mathrm{cm}$ and are prone to slight decrease with the film thicknesses increase. The decrease in resistivity is mainly due to the increase of both carrier density $\mathrm{N}$ and Hall mobility $\mu$. The Hall mobility is mainly influenced by grain boundary scattering, lattice defects and impurity.

In our results, the Hall mobility increase with the increasing film thickness is mainly due to the grain size increase as shown in Fig. 5. Fig. 4b shows the transmittance and absorption properties of films with different thicknesses. Obviously, transmittance is decreasing with the increasing film thickness because of absorption increasing [11].

In addition, it is clear that the plasma resonance wavelength is blue shifted from $1510 \mathrm{~nm}$ to $1450 \mathrm{~nm}$ with the increasing film thickness because of the increasing carrier density which is in agreement with the Hall results. The higher carrier concentration in the thicker films could be attributed to dopant ions (here $\mathrm{Al}^{3+}$ ions) as well as native dopant interstitial zinc atoms and oxygen vacancies $[4,12,13]$.

\subsection{Influence of substrate temperature on the characteristics of AZO films}

Fig. 6a shows that the film deposition rate is slightly decreasing with the substrate temperature increase. This is because at high temperature, sputtered atoms may evaporate from the substrate surface, causing that the growth rate is a little smaller because of the vaporized atoms pumped out at high temperature. The resistivity is first decreasing and then increasing with the substrate temperature as 


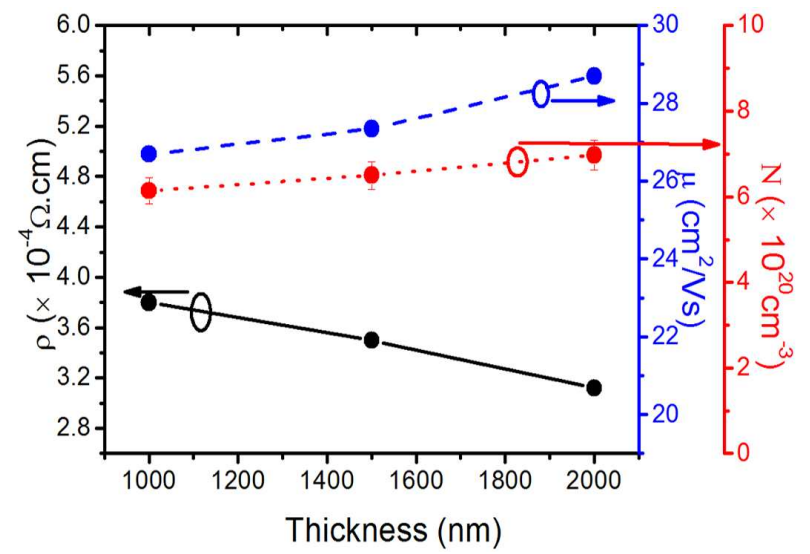

(a)

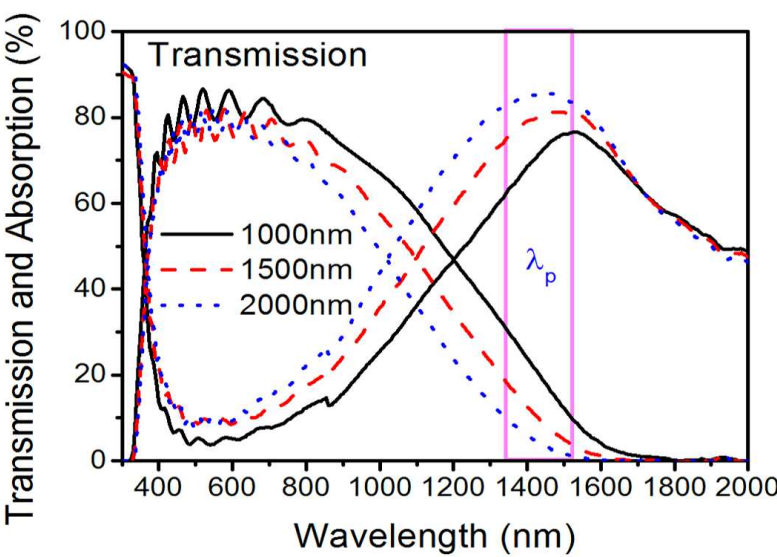

(b)

Fig. 4. (a) Changes in the electrical properties of the films plotted as a function of thin film thickness, (b) Transmittance and absorption of the films with different thicknesses.

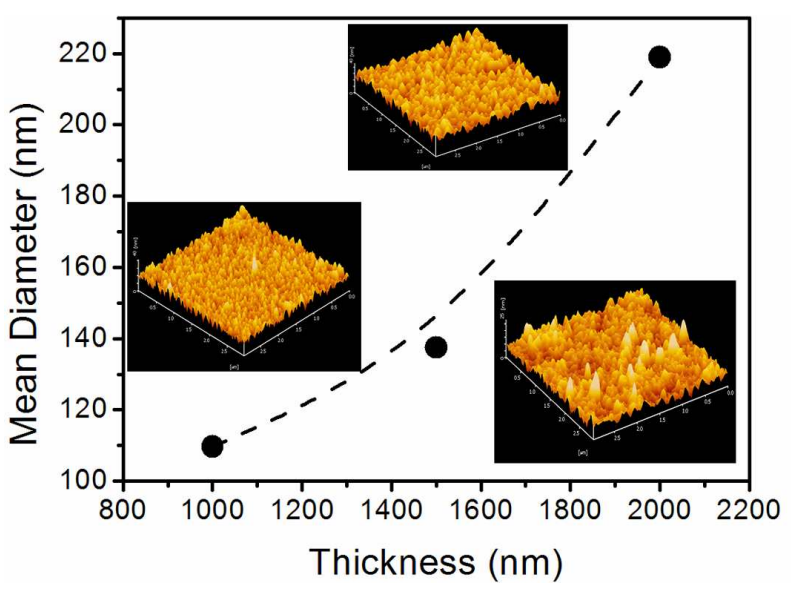

Fig. 5. AFM images of the films as a function of film thickness. shown in Fig. 6a, which is mainly due to the change in Hall mobility as shown in Fig. 6b. Fig. 6b also shows that the carrier density is slightly increasing first and then tends to saturate within the error bars.

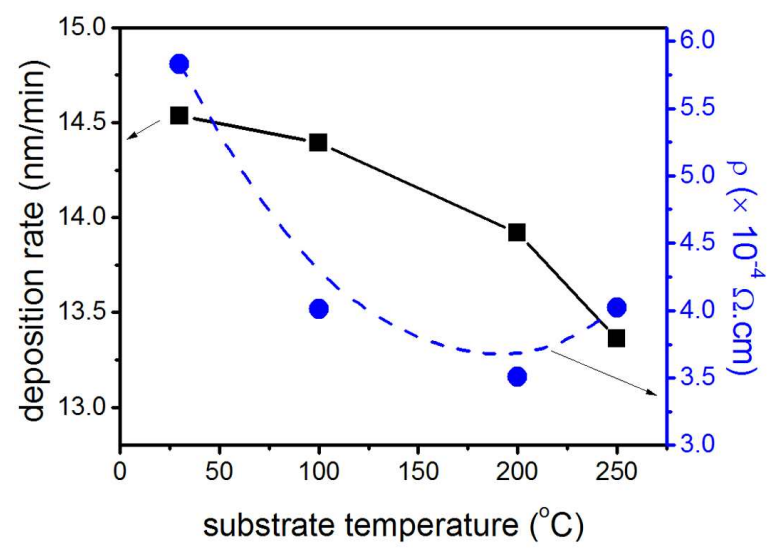

(a)

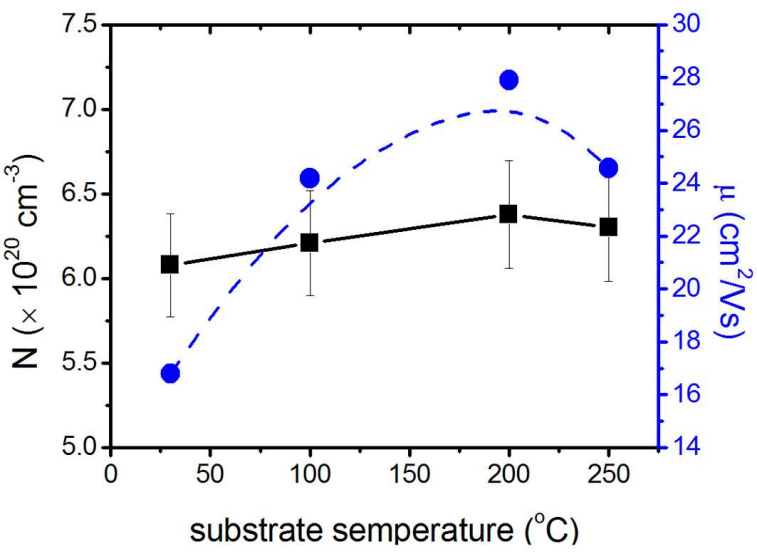

(b)

Fig. 6. (a) Film thickness and resistivity, (b) Hall mobility and carrier density plotted as a function of substrate temperature.

Fig. 7 shows the transmittance, absorption and transmittance edge spectrum of the films deposited at different substrate temperatures. The transparency is increasing with the substrate temperature. The enhancement of optical transmittance may be related to the improvement of film crystallinity with increasing the substrate temperature as evidenced by the decrease in the width of (0 $\left.0 \begin{array}{ll}0 & 2\end{array}\right)$ XRD peak, as shown in Fig. 9. In addition, the plasma absorption peaks are only slightly blue shifted. Such observation confirms that the free 

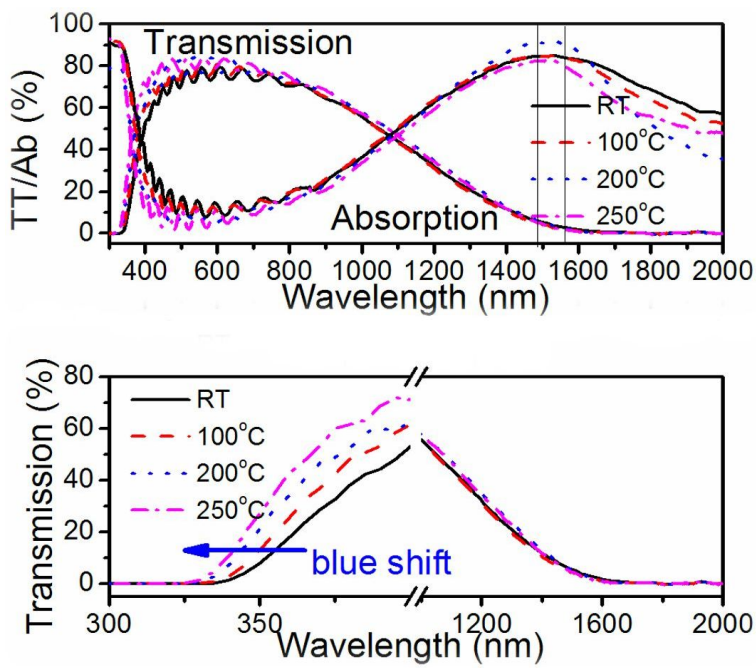

Fig. 7. Transmittance, absorption and transmittance edge of the films deposited at different substrate temperatures.

carrier density shows the same variation trend as it follows from equation 1 . The results of the Hall measurements are the same.

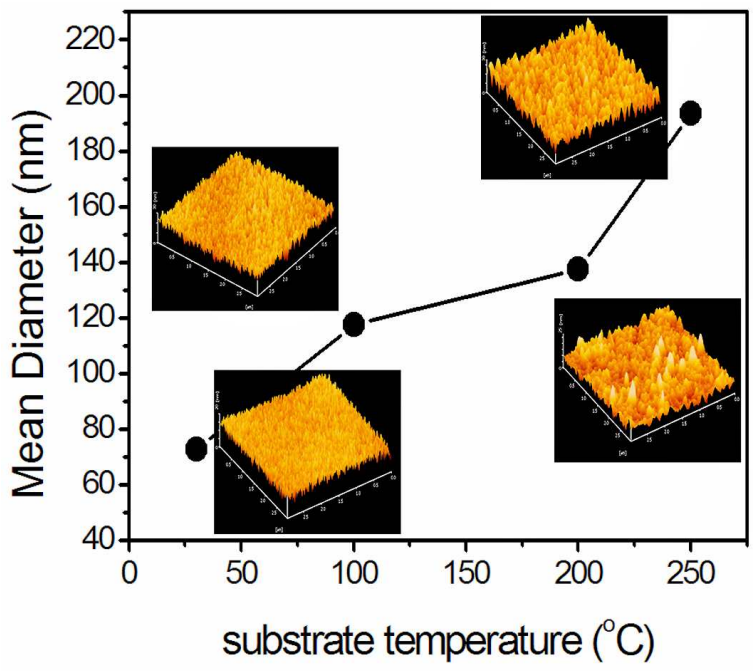

Fig. 8. AFM images of the films prepared at different substrate temperatures.

The carrier densities almost do not change. As we know, the carrier density strongly depends on the doping level. We can conclude that $\mathrm{Al}$ in our study could be doped into the films successfully, even at very low temperature. Of course, the substrate temperature could actually lead to diffusion and evaporation of excessive zinc atoms [13]. Meanwhile, the substrate temperature helps the aluminum atoms to replace the zinc sites and thus excites the donor atoms. Both effects could effectively decrease the defects and thus increase carrier densities. After complete replacing zinc sites by aluminum atoms, the carrier densities become constant.

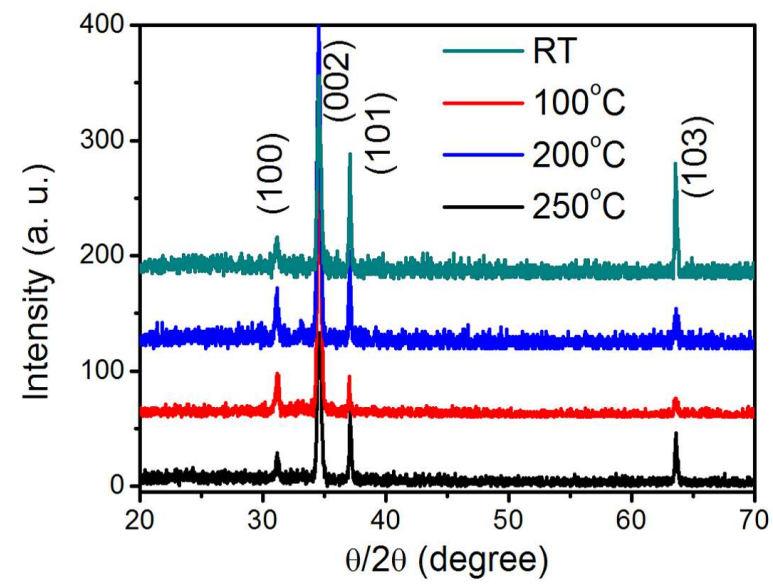

(a)

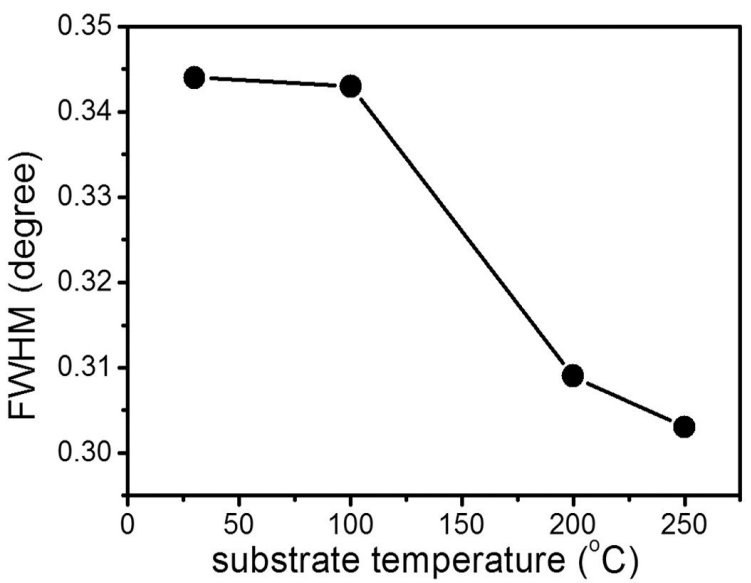

(b)

Fig. 9. (a) XRD and (b) full width at half maximum (FWHM) of (lllll $\left.0 \begin{array}{ll}0 & 2\end{array}\right)$ peak of the films deposited at different substrate temperatures.

The variation of Hall mobility with substrate temperature is a little complicated as shown in Fig. 6b. The initial increase in Hall mobility could be attributed to the increase in the grain size of the films from $70 \mathrm{~nm}$ to $180 \mathrm{~nm}$ with increasing the substrate temperature as shown in Fig. 8, thus 
reducing the grain boundary scattering. When the substrate temperature is higher than $200{ }^{\circ} \mathrm{C}$, the Hall mobility is decreasing. The decrease in Hall mobility may be due to the boundary scattering because of the increase in polycrystalline components. More limitations, such as formation of impurity clusters, higher charge states of ionized donors or extrinsic dopants on interstitial sites, could be possible, as well [14].

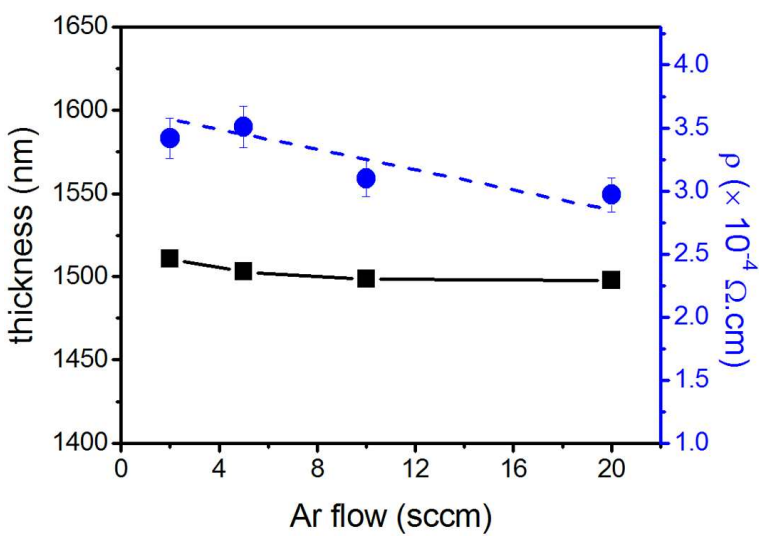

(a)

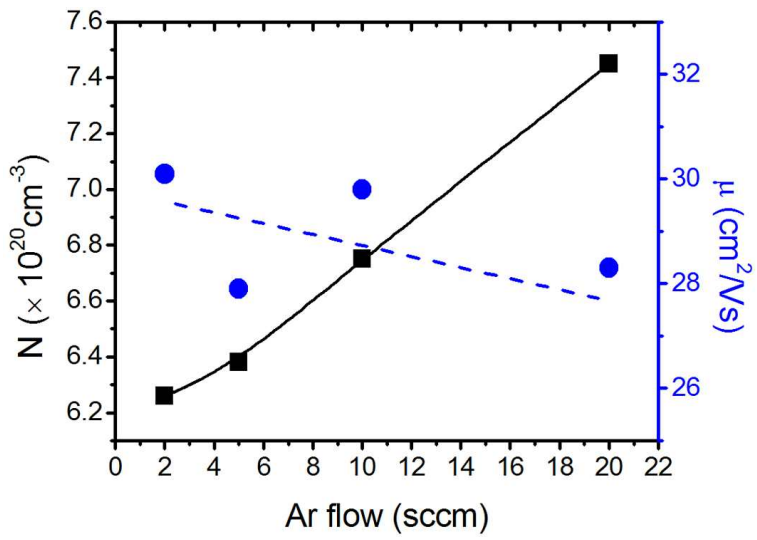

(b)

Fig. 10. (a) Film thickness and resistivity, (b) Hall mobility and carrier density plotted as a function of Ar flow rate.

\subsection{Influence of gas flow rate on the char- acteristics of AZO films}

Fig. 10a shows that the film thicknesses of all films deposited at different Ar flow rates are almost the same. All resistivities are smaller than
$4 \times 10^{-4} \Omega \cdot \mathrm{cm}$, and decrease a little with the $\mathrm{Ar}$ flow rate increase.

Fig. 10b shows the Hall mobility and carrier density determined from the Hall measurement as a function of Ar flow rate. Hall mobilities are slightly decreasing with the Ar flow rate increase, and the carrier densities are increasing with the Ar flow rate increase. The resistivity decrease with the gas flow rate increase is mainly attributed to the carrier density increasing. For the same deposition pressure, at a high Ar flow rate, the pump rate is increasing, thus, the sputtered $\mathrm{O}$ atom lifetime may decrease. The increase in the carrier density at higher gas flow rate may result from an increase in oxygen vacancies.

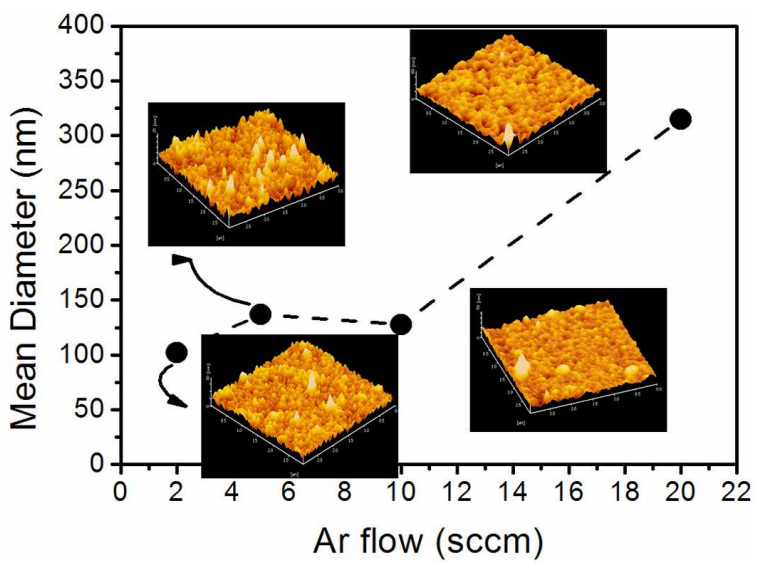

Fig. 11. AFM images of the films prepared at different Ar flow rates.

Fig. 11 shows the AFM images of the films deposited at different gas flow rates. The mean diameter is increasing with the Ar flow rate increase. Such occurrence could be due to the lower deposition rate at the high Ar flow rate. The exact cause is not clear and a further investigation will be carried out in the nearest future. However, the mobility is slightly decreasing with the Ar flow rate increase. Hence, we suggest that the free carrier scattering mechanism governs in the films deposited at different gas flow rates.

Fig. 12 shows the transmission and absorption spectra of the films deposited at different gas flow rates. The transparencies are almost the same at the visible light range, but decrease at NIR region. 
The absorption peak is blue shifted with the Ar flow rate, which is attributed to free carrier density increasing according to equation 1 .

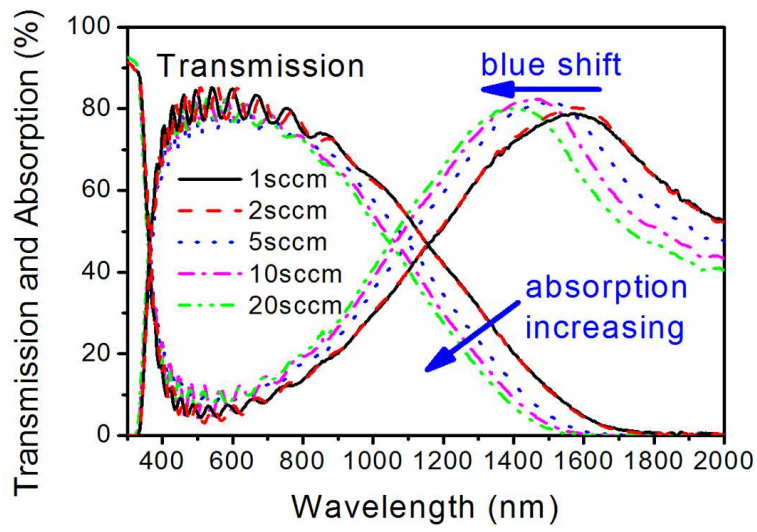

Fig. 12. Transmittance and absorption spectra of the films deposited at different Ar flow rates.

It follows from the above observations that we can get a low resistivity and high transparency AZO film at $200{ }^{\circ} \mathrm{C}$ substrate temperature, low deposition pressure and low Ar flow rate. However, for the application in thin film solar cells, a textured TCO surface is required to provide an effective light trapping. In the following, the AZO films with the thickness of $1500 \mathrm{~nm}$, deposited at $200{ }^{\circ} \mathrm{C}$ substrate temperature and $0.4 \mathrm{~Pa}$ at different Ar flow rates, were etched in $1 \% \mathrm{HCl}$ solution for different durations to get the same sheet resistance of about $3.0 \Omega / \square$.

Fig. 13a shows the total transmission (TT) and diffuse transmission (DT) of the AZO films deposited at different Ar flow rates after etching in $1 \% \mathrm{HCl}$. At the entire wavelength, the etched samples show similar light transmission. However, the etched AZO films display different light scattering properties (light diffusion and haze) as shown in Fig. 13a and Fig. 13b. The film deposited under Ar flow of 10 SCCM shows high diffusion, while the film deposited under Ar flow of 2 SCCM displays relatively low diffusion. These light diffusion properties are mainly correlated with specific surface structures of the films after etching. The corresponding hazes of AZO films after etching increase from $0.35^{\circ}$ to $0.69^{\circ}$ at a wavelength of $600 \mathrm{~nm}$ with the increasing Ar flow rate.

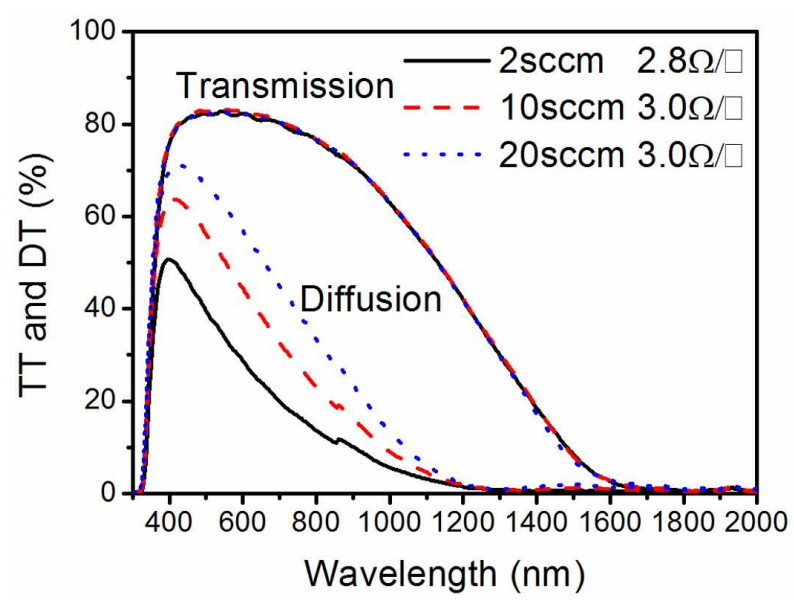

(a)

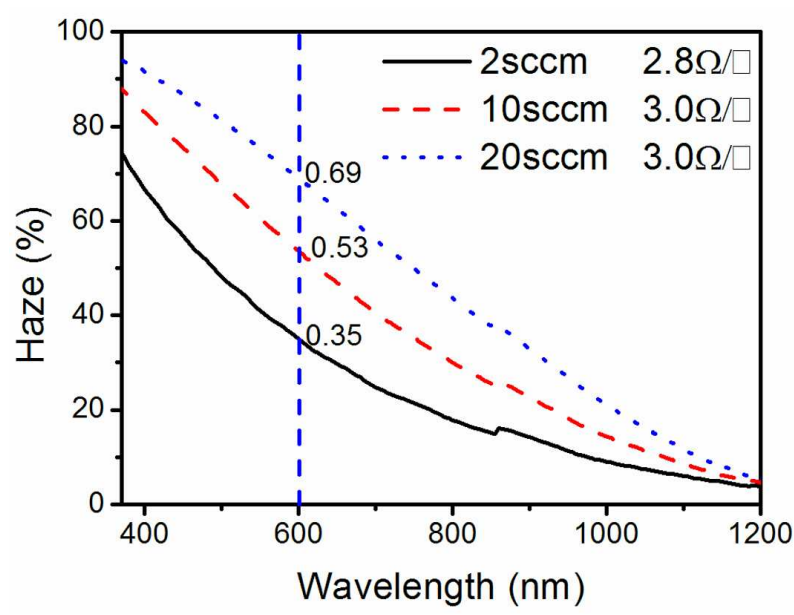

(b)

Fig. 13. Total transmission (TT), diffusion transmission (DT) (a) and haze (b) of AZO films deposited at different Ar flow rates after etching for different durations.

Fig. 14 shows the surface structure of etched AZO films deposited at different Ar flow rates. All the surface textures exhibit a crater-like structure defined by Kluth et al. [10, 15], which leads to an effective light trapping in silicon solar cells. With the increase of Ar flow rate, the feature sizes of the surface structures gradually get larger. It seems that AZO films deposited at higher Ar flow rate become more easily acid-etched. As we know, the etching behavior of AZO films deposited from ceramic targets is determined by deposition parameters, such as the working pressure, substrate temperature, discharge power and $\mathrm{Al}$ doping level $[5,10]$. Apart 

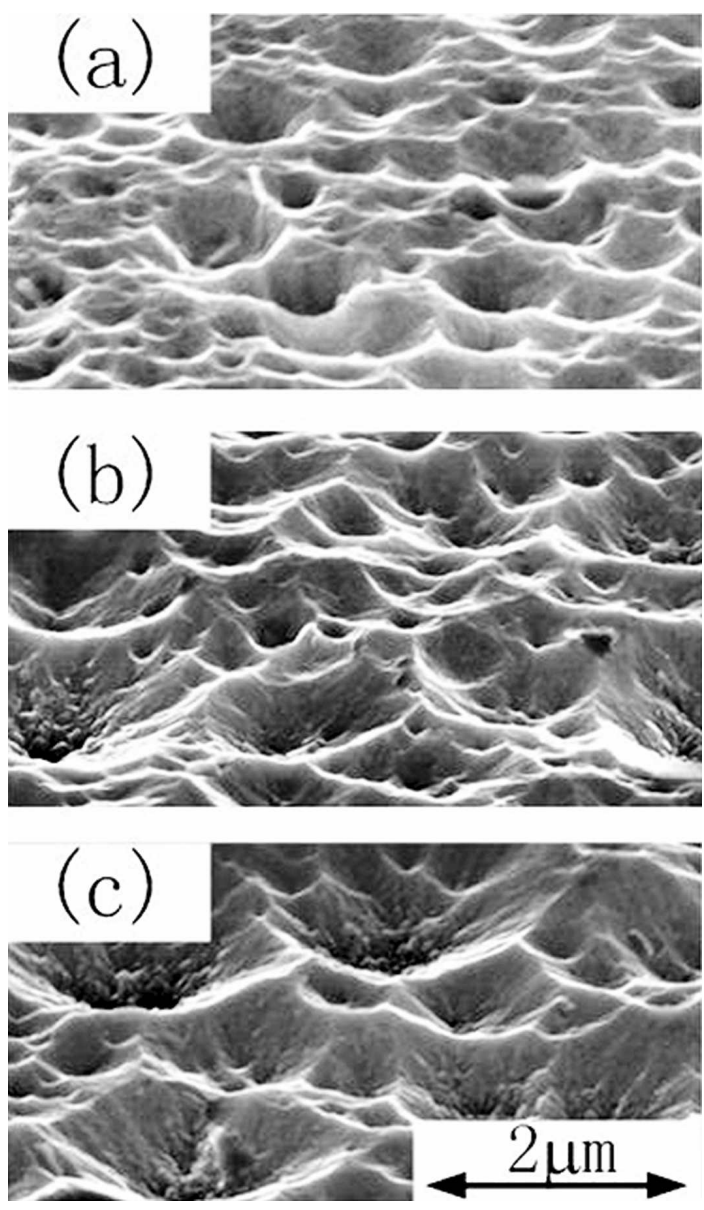

Fig. 14. SEM images of etched AZO films deposited at different Ar flow rates after etching for different durations.

from these, the Ar flow rate is also an important factor affecting the etching behavior.

\section{Conclusions}

The Al-doped $\mathrm{ZnO}$ films were prepared on ultra-clear glass by RF magnetron sputtering using an $\mathrm{AZO}\left(2 \mathrm{wt} \% \mathrm{Al}_{2} \mathrm{O}_{3}\right)$ target. The effects of deposition pressure, films thickness, substrate temperature and Ar flow rate on the electrical and optical properties were discussed in this paper. AFM results showed that the grain mean diameter was increasing with the film thickness, substrate temperature and Ar flow rate. For the films deposited at different pressures and different Ar flow rates, the carrier density determines the film resistivity. For the films deposited at different temperatures, the mobility determines the film resistivity. At $200{ }^{\circ} \mathrm{C}$, low resistivity, smaller than $4 \times 10^{-4} \Omega \cdot \mathrm{cm}$ was obtained, when working pressure was smaller than $0.67 \mathrm{~Pa}$ irrespective of the Ar flow rate. However, higher transparency especially at NIR could be obtained at low Ar flow rate. In addition, all the films showed crater-like surface structures after etching in $1 \% \mathrm{HCl}$. The Ar flow rate is an important factor affecting the etching behavior of Al-doped $\mathrm{ZnO}$ films.

\section{References}

[1] Chopra K.L., Major S., Pandya D.K., Thin Solid Films, 102 (1983), 1.

[2] Agashe C., Kluth O., Schöpe G., Siekmann H., Hüpkes J., RECH B., Thin Solid Films, 442 (2003), 167.

[3] Nomoto J., Konagai M., OKada K., Ito T., Miyata T., Minami T., Thin Solid Films, 518 (2010), 2937.

[4] Minami T., Sato H., Nanto H., Takata S., Jpn. J. Appl. Phys., 24 (1985), L781.

[5] Berginski M., Hüpkes J., Schlute M., Schöpe G., Stiebig H., Wuttig M., J. Appl. Phys., 101 (2007), 074903.

[6] Alam M.J., Cametron D.C., J. Vac. Sci. Technol. A, 19 (2001), 1642.

[7] Tang W., Cameron D.C., Thin Solid Films, 238 (1994), 83.

[8] Bouhssira N., Abed S., Tomasella E., CelLier J., Mosbah A., Aida M.S., JaCQuet M., Appl. Surf. Sci., 252 (2006), 5594.

[9] Agura H., Suzuki A., Matsushita T., Aoki T., OKUdA M., Thin Solid Films, 445 (2003), 263.

[10] Kluth O., Schöpe G., Hüpkes J., Agashe C., MÜller J., RECH B., Thin Solid Films, 442 (2003), 80.

[11] Frank G., Kauer E., Kostlin H., Thin Solid Films, 77 (1981), 107.

[12] Minami T., Nanto H., Takata S., Jpn. J. Appl. Phys., 23 (1984), L280.

[13] Zhu H., Mai Y., Lai B., Wan M., Huang Y., Zhang L., Phys. Status Solidi A 7 (2014), 1589.

[14] Steinhauser J., Fay S., Oliverira N., VallatSauvain E., Ballif C., Appl. Phys. Lett., 90 (2007), 142107.

[15] Kluth O., Rech B., Houben L., Wieder S., Schöpe G., BeneKIng C., WAGner H., LÖFfl A., SCHOCK H.W., Thin Solid Films, 351 (1999), 247.

Received 2016-09-28 Accepted 2017-02-06 